\title{
Building patient-centered care through values assessment integration with advance care planning
}

\author{
Sabrina Q. Mikan, PhD, RN, ACNS-BC, ${ }^{1}$ Cynthia Taniguchi, RN, BSN, OCN, ${ }^{2}$ and \\ J. Russell Hoverman, MD, $\mathrm{PhD}^{3}$ \\ ${ }^{1}$ Texas Oncology, Austin; ${ }^{2}$ McKesson Specialty Health, The Woodlands, Texas; and ${ }^{3}$ Texas Oncology, Dallas
}

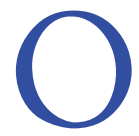
ncologists frequently have to make diagnoses that portend bad outcomes and difficulties in management, among them, for stage IV lung or pancreatic cancer. Many recent studies have shown the importance of appropriate implementation of palliative care and the need for discussing with the patient the goals of treatment early in diagnosis. ${ }^{1-3}$

This process has its challenges. One way to view and meet these challenges is through assessing a patient's personal values regarding his or her life and care. Clinicians (oncologists and midlevel providers) can support a culture of patient and practitioner shared decision making, ensuring that patients with life-limiting illnesses are aware of their choices for end-of-life (EOL) care., Through "values-based" conversations, the clinicians gain perspective of the patients' needs. ${ }^{6}$ This can lead to more formal conversations about EOL care and the completion of advance directive documents.

\section{Advance care planning principles}

With this in mind, clinicians should recognize 5 advance care planning (ACP) principles:

- Discussions are patient-driven and practitioner supported through truthfulness;

- Every patient has a right to have his or her personal values and goals of care known and respected;

- Exploring the benefits and burdens of treatment options is a prerequisite for matching a patient's values and treatment goals;

Manuscript received August 23, 2011; accepted July 12, 2013. Correspondence Sabrina Q. Mikan, PhD, RN, ACNS-BC, Texas Oncology, 6204 Balcones Drive, Austin, TX 78731 (sabrina.mikan@usoncology.com).

Disclosures SM and JRH are affiliated with The US Oncology Network and CT is employed by McKesson Spectialty, which together have designed My Choices, My Wishes ${ }^{\circledR}$, a structured model for ACP education and care delivery.
- Systematic re-evaluation of an individual's change in health status directs the need for further ACP discussions; and

- Specific outcomes metrics are needed to support ACP methodology that is truly beneficial to patients, practitioners, and practices at large. Although the ACP process is not a legal process, an outcome may be development of legal documents, for example, advance directives, living wills, Five Wishes (a patient-friendly legal document that helps adults plan how they want to be cared for if they become seriously ill), physician order for life-sustaining treatment, or donot-resuscitate directives.

\section{Demonstration of a values-based conversation model}

In stage IV lung and pancreatic cancer populations, having a values-based conversation is a critical step because it is important to have documented care directives included within the medical record. How can we facilitate this step? Again, the answer is to learn what is important to patients, given the realities of their life-limiting illnesses. For some individuals, the process may be facilitated by asking them to tell us the level of detail at which they want their health information shared with them or by asking them what a clinician can do for them to support good quality of life during times of disease progression. ${ }^{7,8}$

Rather than the oncologists and patients struggling through difficult conversations and decisions separately, clinicians can facilitate discussion by prompting with statements like, "Tell me what is important to you in your life," and to listen to each patient through ongoing office visits to connect with them on their level, to understand their val-

Commun Oncol 2013:10:209-211 DOI: 10.12788/i.cmonc.0043 (c) 2013 Frontline Medical Communications 
ues, and to help them meet their goals. This allows patients to provide a map of future care for the oncologist. ${ }^{9-11}$ Learning about a patient's health care values provides appropriate insight to individual prioritization of quality of life versus quantity of life and is a major step forward in creating patient-centered care.

\section{Benefits of early ACP}

Through review of current literature, we know that many practitioners are aware of the benefits of ACP. What may be less well known is that early ACP provides distinct advantages in fashioning a plan of care. Earlier intervention allows for greater openness of discussion, with earlier understanding of a patient's values promoting higher levels of comfort and satisfaction for the patient and family during subsequent medical care. ${ }^{12}$ In addition, early ACP conversations diminish barriers to $\mathrm{EOL}$ discussions throughout the continuum of progressive disease, which can lead to effective use of hospice and palliative care, less aggressive care in the last 6 months of life, and more efficient use of practice and patient resources. ${ }^{13}$

From a business perspective, we also know that providing an ACP service adds associated costs through additional training needs, infrastructure requirements and use of staff members on the patient's health care team. Billing has frequently been conducted through oncologists and midlevel providers (MLPs) only, through standard evaluation and management coding for multiple commercial payers and Medicare.

\section{My Choices, My Wishes model for improving uptake of early ACP}

The US Oncology Network is a network of community oncology practices in the United States, consisting of nearly 1,000 physicians who share research, infrastructure, and quality initiatives to improve the quality and value of patient care. Together, The US Oncology Network and McKesson Specialty Health have designed My Choices, My Wishes ${ }^{\circledR}$, a structured model for ACP education and care delivery.

The program was implemented in the Central Austin, Texas Oncology practice, and there has been a measurable impact on patient care as a result of using the program. Over the course of 90 days, the program assisted our practice in identifying 265 unique patients who were clinically appropriate for a values-based conversation. Appointments were scheduled with an MLP who has been trained in having values-based conversations with cancer patients. In our practice model, the MLP may be either an advanced practice nurse or a physician's assistant. Our program is MLP driven, but the counseling appointment

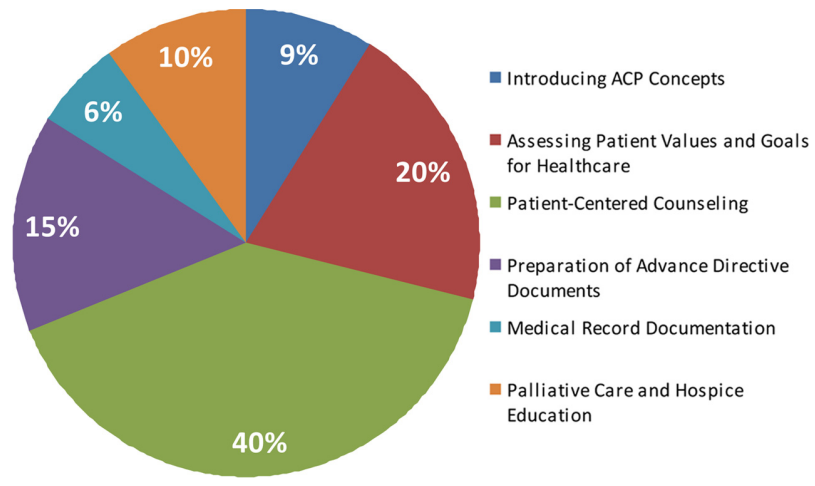

FIGURE 1 Midlevel provider ratio of advanced care planning time spent.

Abbreviation: ACP, advanced care planning.

is interdisciplinary, and we often have a licensed medical social worker (LMSW) present.

Most of the time spent with patients and their family members involves patient-centered counseling (see Figure 1). During counseling, the provider does as little speaking as possible so that the patients can share their values, their goals in life, and discuss needs for living their lives well. "Generous listening" creates the right setting, where the patient and family can hold the discussion among themselves with the MLP and LMSW. It is critical that the MLP guides the conversation, gently providing support and education and empowering the patients to have information to make the best decisions for their care. Other major components of each ACP meeting include assessing a patient's values and goals for health care and assisting in the preparation of advance directive documents.

As the conversation unfolds, the topics of palliative care and hospice may be addressed. In this discussion, basic education is reviewed so that the patients and their families are aware of services that can ultimately improve their quality of life and symptom management.

Each week, Austin Central providers review a report of eligible patients with primary oncologists so that each is aware that advance care planning services can be provided for their patients. We hold interdisciplinary meetings, MLP and LMSW, with patients that prove to be very beneficial to both the patients and their families.

It is important to note that nursing and social work disciplines hear different topics when a person is sharing his or her story, values, and needs. It is a privilege to sit and talk with patients who are facing an uncertain time in their lives, and to empower them to state what their values are, so that they (along with the oncologist) can "map" their wishes and goals for health care they will receive. In addition, should the patient have an interest in selecting their medical power of attorney or completing an advance 
directive, we can discuss who might best serve them in that capacity. We can also discuss what life-sustaining interventions the patient would or would not want in situations of declining health. Some of these life sustaining interventions require educating the patient and family about what may, or may not, be helpful for someone with life-limiting illness. At first, the interaction itself can be daunting for the patient, but as the conversation unfolds, they relax and begin to take the lead in what they want to talk about and focus on their personal preferences for care.

At times, the provider can see the patient's expressions change when introducing the topic of ACP. It is important to assure them with security that they are having this conversation not during a time of crisis, but at a time when they are able to make sound decisions and share those decisions with their family and health care providers. Each patient agrees that this is a focus they've not had in their care, but know the importance of sitting and taking the time to begin the conversation. The patient may list various symptoms or personal issues, such as income and investments, and the MLP and LMSW can listen and begin to process and offer support.

Should symptoms be a topic that they report, an introduction to palliative care is provided. If needed, the patient is offered an outpatient palliative care referral to our palliative care clinic. As the conversation is ending, the patient and family are aware that this is just the beginning of their conversations and that they can feel comfortable talking about the things in their lives that are important for them to live well. Most are relaxed by the end and will often comment that the exchange "wasn't so bad. I thought it was going to be very difficult. I feel good. Thank you!" Ultimately, providing this service has an impact on satisfaction for both patient and provider. The shared experience enables a forum for patient-centered care and trust, and patients understand through this meaningful discussion that they are valued.

\section{Program scalability}

My Choices, My Wishes is designed for program scaling to fit practice needs and resources. Practices begin by focusing on 2 diagnoses: stage IV lung and pancreas cancers. Additional program integration occurs over time, as clinicians become more comfortable with the program, patient interactions, and program administration. Program infrastructure is fostered through the use of the McKesson Specialty Health iKnowMed electronic health record system, which allows community practitioners to identify patients within the practice who are clinically appropriate for advance care planning discussions and to access, record, and share data on components of care. All levels of program engagement include assessment of patient values and goals for health care, intervention documentation tools, and defined metrics for evaluating program outcomes and performance.

\section{Conclusion}

My Choices, My Wishes is aimed at systematically integrating advance care planning activity early into the cancer experience by enlisting involvement of patients, MLPs, and oncologists. The program provides structured EOL support that includes education to improve health literacy, methodology for assessing patient values and goals for care, and it creates an environment for care planning that matches each patient's specific goals. Because we recognize the importance of these discussions being directed by patient values, we are currently piloting and validating a patient values and goals for health care instrument. Ongoing studies will assess how this overall strategy for integrating and implementing advance care planning affects quality of care, quality of life, health care resource use, and cost.

\section{References}

1. Mack JW, Conin A, Keathing NL, et al. Associations between end-of-life discussion characteristics and care received near death: a prospective cohort study. J Clin Oncol. 2012;30:4387-4395.

2. Hermann C, Looney S. Determinants of quality of life in patients near the end of life: a longitudinal perspective. Oncol Nurs Forum. 2011;38(1):23-31.

3. Detering K, Hancock A, Reade M, Silvester W. The impact of advance care planning on end of life care in elderly patients: randomized controlled trial. $\mathrm{Br}$ Med J. 2010;340:c1345.

4. Weeks JC, Catalano PJ, Cronin A, et al. Patients' expectations about effects of chemotherapy for advanced cancer. NEJM. 2012; 367:1616-1625.

5. Braun U, McCullough L. Preventing life-sustaining treatment by default. Ann Fam Med. 2011;9(3):250-256.

6. Wright AA, Zhang B, Ray A, et al. Associations between end-oflife discussion, patient mental health, medical care near death and caregiver bereavement adjustment. JAMA. 2008;300:1665-1673.

7. Smith T, Temin S, Alesi E, et al. American Society of Clinical Oncology provisional clinical opinion: The integration of palliative care into standard oncology care. J Clin Oncol. 2011;30(8): 880-887.

8. Temel JS, Greer JA, Muzikansky A, et al. Early palliative care for patients with metastatic non-small-cell lung cancer. $N$ Engl J Med. 2010;363:733-742.

9. Earle CC. It takes a village. J Clin Oncol. 2012;30:353-354.

10. Kass-Bartelmes B, Hughes R. Advance care planning: preferences for care at the end of life. Rockville (MD): Agency for Healthcare Research and 10. Quality. Research in Action, 12. AHRQPub No. 03-0018, 2003.

11. Lundquist G, Rasmussen BH, Axelsson B. Information of imminent death or not: does it make a difference? J Clin Oncol. 2011;29:3927-3931.

12. Keating N, Landrum M, Rogers S, et al. Physician factors associated with discussions about end-of-life care. Cancer. 2010;116: 998-1005.

13. Dudgeon DJ, Knott C, Chapman C, et al. Development, implementation, and process evaluation of a regional palliative care quality improvement project. J Pain Symptom Manage. 2009;38: 483-495. 